\title{
Technè
}

La science au service de l'histoire de l'art et de la préservation des biens culturels

47 | 2019

Bernard Palissy : nouveaux regards sur la céramique française auX $\mathrm{XVI}{ }^{\mathrm{e}}$ et $\mathrm{XVII}{ }^{\mathrm{e}}$ siècles

\section{Chalcis ou non? Recontextualiser des plats byzantins conservés dans un musée}

Chalcis or not? Re-contextualising Byzantine ceramics kept in a museum

Jacques Burlot, Yona Waksman, Anne Bouquillon et Laurence Tilliard

\section{OpenEdition}

1 Journals

Édition électronique

URL : http://journals.openedition.org/techne/1635

DOI : 10.4000/techne. 1635

ISSN : 2534-5168

Éditeur

C2RMF

Édition imprimée

Date de publication : 1 juin 2019

Pagination : 150-157

ISBN : 978-2-11-152830-7

ISSN : $1254-7867$

Référence électronique

Jacques Burlot, Yona Waksman, Anne Bouquillon et Laurence Tilliard, «Chalcis ou non?

Recontextualiser des plats byzantins conservés dans un musée », Technè [En ligne], 47 | 2019, mis en ligne le 01 juin 2020, consulté le 23 juillet 2020. URL : http://journals.openedition.org/techne/1635 ; DOI : https://doi.org/10.4000/techne.1635

\section{(c) $98 \Theta$}

La revue Technè. La science au service de l'histoire de l'art et de la préservation des biens culturels est mise à disposition selon les termes de la Licence Creative Commons Attribution - Pas d'Utilisation Commerciale - Pas de Modification 4.0 International. 

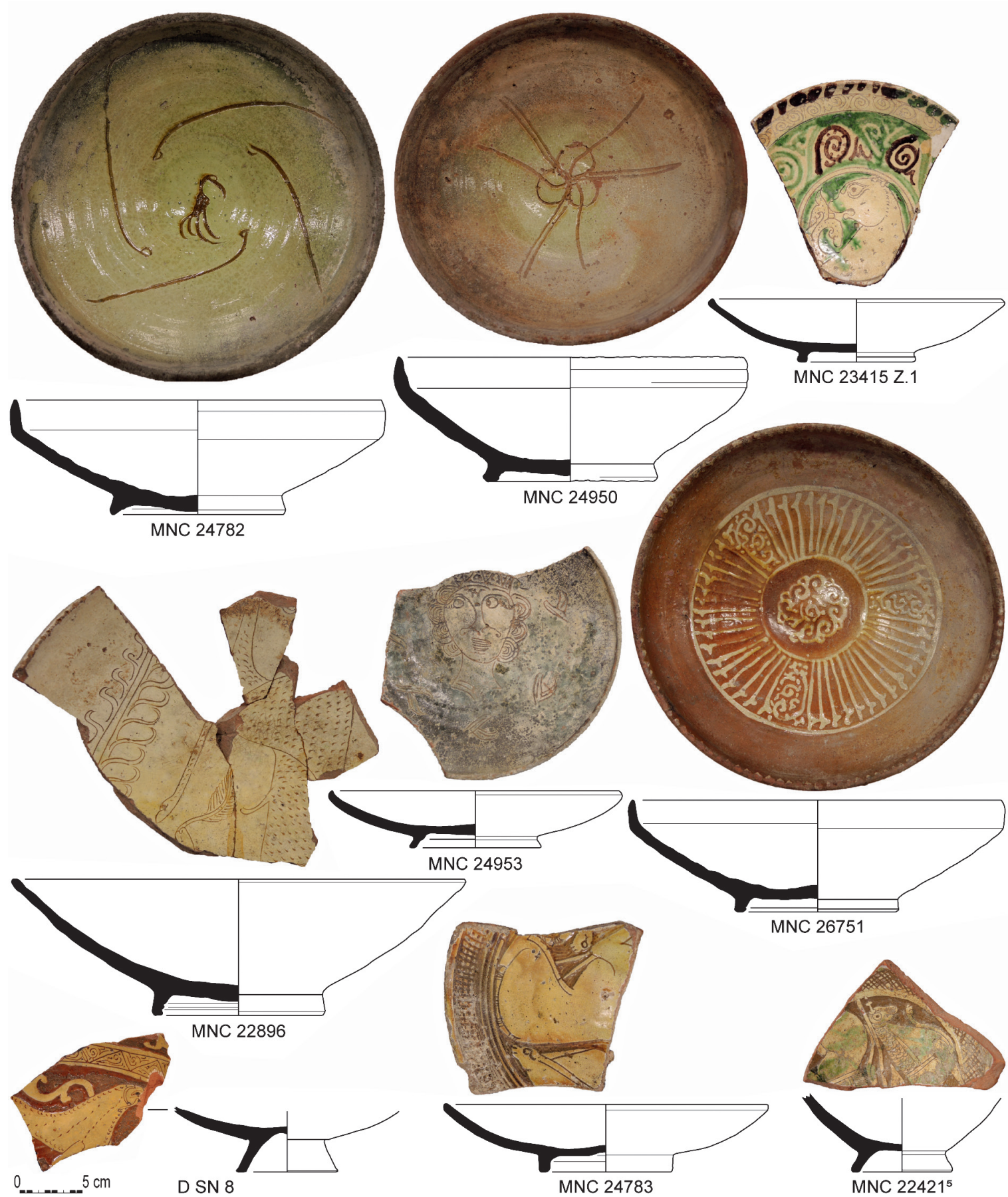

MNC 24953

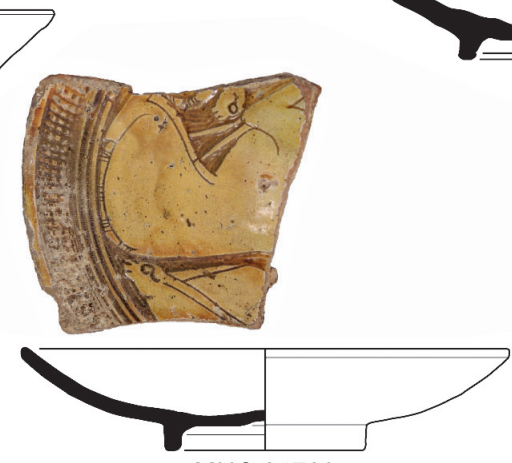

MNC 26751

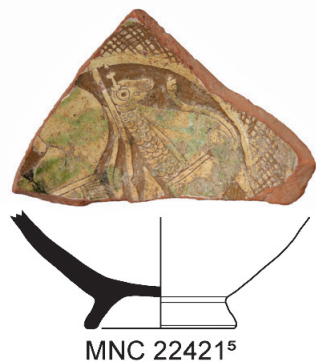

Fig. 1. Pièces analysées, issues des collections du musée national de Céramique de Sèvres, et représentant différents types de céramiques byzantines.

๑) S. Y. Waksman (photos) ; S. Y. Waksman, J. Burlot, C. Richarté (dessins) ; F. Guériel, J. Burlot (DAO). 
Jacques Burlot

Yona Waksman

Anne Bouquillon

Laurence Tilliard

\section{Chalcis ou non ? Recontextualiser des plats byzantins conservés dans un musée}

Chalcis or not? Re-contextualising Byzantine ceramics kept in a museum
Résumé. La comparaison des compositions chimiques des pâtes constitutives de neuf plats byzantins, conservés dans les collections du musée national de Céramique de Sèvres, avec celles de pièces archéologiques de référence, permet de réintégrer la plupart des xuvres dans l'ensemble de la production de Chalcis des XII et XIII ${ }^{e}$ siècles. Pour deux pièces, en revanche, se pose la question de l'authenticité. Les perturbations géochimiques liées à un séjour prolongé des pièces en contexte marin sont également discutées et une méthodologie spécifique est proposée.

Mots-clés. Céramique byzantine, provenance, techniques de fabrication, analyses PIXE, AGLAE, épaves, altérations marines.
Abstract. The comparison of chemical compositions of ceramic bodies of nine Byzantine plates kept in the Musée national de céramique, Sèvres, with those of reference archaeological samples enabled us to associate most of the plates to the 12th-13th century production of Chalcis. Two pieces, however, raise the question of authenticity. Geochemical perturbations related to prolonged immersion in a marine environment are also discussed and a specific methodology is proposed.

Keywords. Byzantine ceramics, provenance, manufacturing techniques, PIXE, AGLAE analyses, shipwrecks, alterations in marine environments.

\section{Introduction}

Les collections de céramiques byzantines de nombreux musées à travers le monde sont surtout constituées de grands plats datés des $\mathrm{XII}^{\mathrm{e}}$-XIII ${ }^{\mathrm{e}}$ siècles qui provenaient vraisemblablement d'épaves ${ }^{1}$. Ils se rattachent à différents types dont la dénomination est liée à leur technique de décoration : "Fine Sgraffito Ware”, "Incised Sgraffito Ware”, "Champlevé Ware”, etc. Ces types de céramiques sont découverts en Méditerranée et en mer Noire, notamment dans les grands ports, de Marseille à Saint-Jean d'Acre, de Chersonèse à Paphos. Des recherches archéologiques et archéométriques ont été menées ces dernières années sur une centaine d'exemplaires issus des fouilles de plusieurs sites, et d'une épave en mer Égée. L'analyse chimique et minéralogique de leurs pâtes, ainsi que l'étude des sources d'argiles potentielles ont montré qu'ils correspondent à une production unique, la "Main Middle Byzantine Production" (MBP), dont les ateliers étaient localisés à Chalcis (Grèce) ${ }^{2}$.

Dans les musées, les informations sur les contextes archéologiques de découverte sont limitées voire inexistantes, même si la présence de cristallisations de sels visibles sur la surface indique du matériel provenant d'épave. Parmi les collections de céramiques byzantines du musée national de Céramique de Sèvres, plusieurs plats pourraient être dans ce cas et relèvent typologiquement de la production de Chalcis. Nous en avons sélectionné sept qui étaient parmi les plus représentatifs, ainsi que deux autres pièces dont l'attribution à Chalcis paraissait plus douteuse. Tenter de retrouver leur identité, de les recontextualiser, est donc d'un intérêt évident. Notre étude se propose de le faire sur ces neuf pièces en comparant les caractéristiques de leur matériau avec celles d'un corpus d'échantillons archéologiques de provenance, d'origine et de datation connues. Elle a aussi pour objectif de définir leurs techniques de fabrication par l'analyse des glaçures.

Cette étude s'inscrit par ailleurs dans la continuité d'une vaste enquête archéométrique portant aussi sur les collections de céramiques byzantines du musée du Louvre ${ }^{3}$.

Nous devions mettre en œuvre des techniques totalement non destructives. Cette exigence nous a conduit à réaliser des analyses par PIXE en faisceau extrait à l'accélérateur AGLAE, 
et à mettre en place une méthodologie adaptée au matériel d'épaves.

\section{Pièces analysées}

Neuf pièces des collections du musée national de Céramique de Sèvres ont été analysées (fig. 1). Trois d'entre elles sont des plats complets qui font partie de l'exposition permanente, mentionnés dans l'inventaire du musée comme provenant d'une épave entre les eaux territoriales grecques et turques. Leur dépôt en milieu marin est déduit de la présence de concrétions au revers de l'un des plats, le revers d'un autre ayant probablement été gratté. Deux d'entre eux (MNC 24782, MNC 24950) présentent des décors typiques de la MBP, d'incisions larges ("Incised Sgraffito Ware") similaires à des pièces trouvées dans l'épave de Kastellorizo ${ }^{4}$. Le troisième présente un décor épigraphique d'engobe ("Slip-Painted Ware”, MNC $26751^{5}$ ). Les autres pièces (MNC 22421 MNC 22896, MNC 24953, MNC 23415 Z.1, MNC 24783, D SN 8) sont de grands tessons enregistrés en tant que céramique byzantine ou "poterie musulmane », qui relèvent d'autres types ("Painted Fine Sgraffito", "Champlevé Ware”). La question de leur authenticité se posait pour au moins deux d'entre eux, l'un stylistiquement assez inhabituel (MNC 24953), l'autre portant la mention «copie»(D SN 8).

Cinq tessons archéologiques de $\mathrm{MBP}^{6}$ ont également été analysés pour compléter la caractérisation des glaçures et en tant que matériel comparatif. Ces tessons, attribués à Chalcis

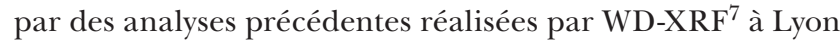
(CNRS UMR 5138), provenaient pour deux d'entre eux de l'épave de Kavalliani (Grèce), découverte lors d'une prospection sous-marine menée par l'Institut hellénique d'archéologie marine et l'Éphorie des antiquités sous-marines d'Athènes $\left(\right.$ BZY872, BZY873) ${ }^{8}$, les trois autres provenant de fouilles de sauvetage menées par la 23e Éphorie des antiquités byzantines à Thèbes et à Chalcis (BZY521, BZY533, BZY754) ${ }^{9}$. Ils relèvent des mêmes types associés à la MBP (BZY521 et BZY872 : "Incised Sgraffito Ware" ; BZY533 : "Painted Fine Sgraffito"; BZY754 : "Green and Brown Painted Ware" ; BZY873 : "SlipPainted Ware").

\section{Analyses des pâtes}

\section{Méthodologie et protocoles analytiques}

Nous avons dû aborder les analyses en tenant compte de deux paramètres limitatifs : l'obligation de faire des analyses de surface sans prélèvement d'une part et, d'autre part, la modification des compositions chimiques de la pâte céramique quand elle est en contact avec un milieu marin. Les analyses ont été réalisées par PIXE en faisceau extrait ${ }^{10}$, soit de façon non destructive sur la surface des pièces de musée et des échantillons archéologiques BZY872 et BZY873, soit sur coupe stratigraphique, enrobée dans une résine époxy et polie, s'agissant des autres échantillons qui servaient de références pour la production de Chalcis. Les analyses de tessons archéologiques permettaient d'évaluer les écarts de résultats entre différentes méthodes (PIXE et WD-XRF), protocoles analytiques (en surface ou sur coupe), et conditions d'enfouissement (milieu terrestre ou marin) et de conservation (tableau 1). Les données prises comme référence pour la production de Chalcis sont les analyses réalisées à Lyon par WD-XRF sur perles, portant sur des céramiques archéologiques provenant de contextes terrestres (tableau 2) ${ }^{11}$.

Influence des différences de protocoles analytiques Une première comparaison portant sur les échantillons archéologiques analysés en coupe (BZY521, BZY533, BZY754) indique que les données obtenues par PIXE, avec ce protocole, restent relativement proches de celles obtenues par WD-XRF ${ }^{12}$. En revanche, les compositions des échantillons analysés en surface (BZY872, BZY873), c'est-à-dire dans les mêmes conditions que les pièces de musée, peuvent être proches de celles obtenues par WD-XRF (BZY873), ou en revanche s'en écarter de façon assez marquée (jusqu'à $30 \%$ d'écart relatif, BZY872). Ce dernier point donne une première indication sur les limites d'une comparaison directe entre des données PIXE, en analyse de surface dans des conditions non destructives, et des données de référence WD-XRF.

\section{Influence du contexte marin}

Ces limitations sont renforcées par des phénomènes d'altération en milieu marin. Ce dernier génère en effet des modifications chimiques dans les matériaux qui composent les céramiques, du fait d'échanges avec l'eau de mer ainsi que de l'activité bactériologique ${ }^{13}$. Certains échantillons témoignent d'altérations marines typiques (tableau 1), qui avaient déjà été observées par analyses WD-XRF pour des plats provenant de l'épave de Kavalliani ${ }^{14}$.

Les teneurs en $\mathrm{MgO}$ sont ainsi plus élevées pour les échantillons probablement issus d'un milieu marin avec des valeurs supérieures à 3,3\% et qui peuvent dépasser $8 \%$, alors qu'elles sont inférieures à 2,3\% pour les échantillons issus de contextes terrestres. L'augmentation du magnésium dans les compositions de céramiques issues de milieu marin est un phénomène déjà bien attesté et témoigne d'une fixation de cet élément pouvant être attribuée à l'apparition de phases carbonatées comme l'hydrotalcite ${ }^{15}$. À l'inverse, leurs teneurs en $\mathrm{K}_{2} \mathrm{O}$ sont plus faibles, résultat possible d'une dissolution de carbonates potassiques par un renouvellement continu d'eau ${ }^{16}$. Enfin, des teneurs élevées en soufre sont aussi détectées dans certains cas, qui pourraient être liées à la formation de sulfates, tels que le gypse, en milieu marin ${ }^{17}$.

Ces tendances sont observées pour la majorité des pièces de musée, à l'exception de deux d'entre elles (MNC 22896 et MNC $22421^{5}$ ). Ces dernières présentent des teneurs en $\mathrm{MgO}$ et $\mathrm{K}_{2} \mathrm{O}$ proches des valeurs de référence. Même si l'une de ces 
Tableau 1. Compositions élémentaires des pâtes - données brutes des analyses PIXE et WD-XRF (en pourcentage massique d'oxyde ; ld : valeur inférieure à la limite de détection ; nd : non déterminé).

\begin{tabular}{|c|c|c|c|c|c|c|c|c|c|c|c|c|c|}
\hline & Échantillon & $\mathrm{CaO}$ & $\mathrm{Fe}_{2} \mathrm{O}_{3}$ & $\mathrm{TiO}_{2}$ & $\mathrm{~K}_{2} \mathrm{O}$ & $\mathrm{SiO}_{2}$ & $\mathrm{Al}_{2} \mathrm{O}_{3}$ & $\mathrm{MgO}$ & MnO & $\mathrm{Na}_{2} \mathrm{O}$ & $\mathrm{P}_{2} \mathrm{O}_{5}$ & $\mathrm{SO}_{3}$ & $\mathrm{Cl}$ \\
\hline \multicolumn{14}{|c|}{ PIÈCES DE MUSÉES } \\
\hline \multicolumn{14}{|l|}{ Surface } \\
\hline & MNC 24782 & 3.80 & 9.37 & 0.80 & 3.22 & 51.70 & 19.61 & 4.24 & 0.11 & 3.44 & 0.20 & 0.56 & 2.90 \\
\hline & MNC 26751 & 5.56 & 7.70 & 1.00 & 2.89 & 51.34 & 20.03 & 6.50 & 0.12 & 1.25 & 0.13 & 1.86 & 1.61 \\
\hline & MNC 24950 & 3.28 & 8.66 & 1.07 & 2.79 & 49.30 & 22.01 & 8.03 & 0.11 & 1.17 & 0.25 & 1.63 & 1.63 \\
\hline & MNC 224215 & 6.20 & 6.58 & 0.74 & 3.51 & 56.72 & 19.25 & 3.11 & 0.13 & 1.38 & 0.29 & 2.07 & 0.02 \\
\hline & MNC 22896 & 5.34 & 7.57 & 0.85 & 3.71 & 57.70 & 20.34 & 2.28 & 0.13 & 1.32 & 0.21 & 0.54 & 0.01 \\
\hline & MNC 24953 & 10.36 & 7.46 & 1.08 & 2.81 & 46.52 & 19.92 & 8.10 & 0.09 & 1.13 & 0.10 & 1.82 & 0.49 \\
\hline & MNC 23415 Z.1 & 6.62 & 8.08 & 0.70 & 2.60 & 57.14 & \begin{tabular}{|l|}
17.18 \\
\end{tabular} & 3.72 & 0.16 & 1.36 & 1.05 & 0.74 & 0.10 \\
\hline & MNC 24783 & 5.70 & 8.58 & 0.99 & 2.60 & 47.75 & 22.72 & 7.42 & 0.13 & 1.27 & 0.12 & 1.14 & 1.11 \\
\hline & D SN 8 & 11.05 & 7.82 & 0.85 & 2.41 & 56.84 & 14.55 & 4.17 & 0.17 & 1.08 & 0.24 & 0.48 & 0.31 \\
\hline \multicolumn{14}{|c|}{ ÉCHANTILLONS ARCHÉOLOGIQUES } \\
\hline \multicolumn{14}{|l|}{ Surface } \\
\hline & BZY872 & 6.79 & 8.60 & 1.14 & 2.57 & 53.40 & 22.49 & 3.35 & 0.09 & 1.06 & 0.19 & 0.31 & 0.01 \\
\hline \multirow[t]{2}{*}{ LYON-XRF } & BZY872 & 5.14 & 7.35 & 0.901 & 3.19 & 58.55 & 19.59 & 3.27 & 0.1165 & 1.55 & 0.16 & nd & nd \\
\hline & BZY873 & 5.45 & 6.90 & 0.85 & 2.91 & 58.40 & 19.54 & 4.18 & 0.12 & 1.20 & 0.23 & 0.21 & $<l d$ \\
\hline LYON-XRF & BZY873 & 5.54 & 7.00 & 0.882 & 3.03 & 58.50 & 18.86 & 4.18 & 0.1099 & 1.56 & 0.16 & nd & nd \\
\hline \multicolumn{14}{|l|}{ Coupe } \\
\hline & BZY754 & 7.39 & 6.88 & 1.03 & 3.16 & 57.68 & 19.68 & 1.89 & 0.12 & 1.10 & 0.51 & $<$ ld & 0.03 \\
\hline \multirow[t]{2}{*}{ LYON-XRF } & BZY754 & 6.18 & 7.11 & 0.854 & 3.58 & 58.90 & 19.11 & 2.27 & 0.1158 & 1.32 & 0.32 & nd & nd \\
\hline & BZY533 & 5.55 & 7.40 & 0.87 & 3.71 & 58.66 & 20.29 & 2.31 & 0.12 & 0.89 & 0.15 & 0.03 & 0.02 \\
\hline \multirow[t]{2}{*}{ LYON-XRF } & BZY533 & 5.23 & 7.95 & 0.877 & 3.83 & 56.65 & 21.04 & 2.64 & 0.1234 & 1.27 & 0.16 & nd & nd \\
\hline & BZY521 & 5.01 & 6.85 & 0.84 & 3.52 & 61.24 & 19.19 & 1.95 & 0.11 & 1.06 & 0.21 & $<$ ld & 0.54 \\
\hline LYON-XRF & BZY521 & 5.21 & 7.59 & 0.854 & 3.69 & 58.26 & 20.13 & 2.46 & 0.1192 & 1.23 & 0.15 & nd & nd \\
\hline
\end{tabular}

Tableau 2. Compositions élémentaires des pâtes - données renormalisées des analyses PIXE des pièces de musée, et données comparatives du groupe de référence de Chalcis obtenues par analyses WD-XRF (en pourcentage massique d'oxyde). Pour les pièces de musée, la normalisation à $100 \%$ a été effectuée sur les éléments majeurs et mineurs, sans intégrer les éléments $\mathrm{S}$ et $\mathrm{Cl}$, et en forçant la valeur de $\mathrm{MgO}$ à $2,5 \%$.

\begin{tabular}{|l|l|l|l|l|l|l|l|l|l|l|}
\hline Échantillon & $\mathrm{CaO}^{\prime}$ & $\mathrm{Fe}_{2} \mathrm{O}_{3}$ & $\mathrm{TiO}_{2}$ & $\mathrm{~K}_{2} \mathrm{O}$ & $\mathrm{SiO}_{2}$ & $\mathrm{Al}_{2} \mathrm{O}_{3}$ & $\mathrm{MgO}$ & $\mathrm{MnO}$ & $\mathrm{Na}_{2} \mathrm{O}$ & $\mathrm{P}_{2} \mathrm{O}_{5}$ \\
\hline \multicolumn{8}{|c|}{ Pièces de musées (analyses en surface) } \\
\hline MNC 24782 & 4.02 & 9.91 & 0.84 & 3.40 & 54.64 & 20.72 & 2.50 & 0.12 & 3.64 & 0.21 \\
\hline MNC 26751 & 6.03 & 8.34 & 1.08 & 3.13 & 55.61 & 21.70 & 2.50 & 0.13 & 1.35 & 0.14 \\
\hline MNC 24950 & 3.52 & 8.61 & 1.20 & 3.00 & 54.73 & 24.73 & 2.50 & 0.11 & 1.33 & 0.28 \\
\hline MNC 224215 & 6.38 & 6.77 & 0.76 & 3.61 & 58.34 & 19.79 & 2.50 & 0.13 & 1.42 & 0.30 \\
\hline MNC 22896 & 5.36 & 7.60 & 0.85 & 3.72 & 57.89 & 20.41 & 2.50 & 0.13 & 1.33 & 0.21 \\
\hline MNC 24953 & 11.29 & 8.13 & 1.18 & 3.06 & 50.69 & 21.70 & 2.50 & 0.10 & 1.23 & 0.11 \\
\hline MNC 23415 Z.1 & 6.80 & 8.30 & 0.72 & 2.67 & 58.72 & 17.65 & 2.50 & 0.16 & 1.40 & 1.08 \\
\hline MNC 24783 & 6.18 & 9.31 & 1.07 & 2.83 & 51.80 & 24.65 & 2.50 & 0.14 & 1.38 & 0.13 \\
\hline D SN 8 & 11.34 & 8.02 & 0.88 & 2.47 & 58.32 & 14.93 & 2.50 & 0.18 & 1.11 & 0.25 \\
\hline \multicolumn{10}{|c|}{ Groupe de référence de Chalcis léchantillons archéologiques, n=56, Waksman et al. 2014) } \\
\hline m & 5.17 & 7.38 & 0.848 & 3.70 & 58.68 & 19.68 & 2.52 & 0.1190 & 1.40 & 0.19 \\
\hline$\sigma$ & 0.62 & 0.42 & 0.014 & 0.21 & 1.90 & 0.98 & 0.18 & 0.0062 & 0.17 & 0.05 \\
\hline
\end{tabular}


pièces présente des teneurs en soufre élevées (environ $2 \%$ $\mathrm{SO}_{3}$ ), on peut supposer qu'elles ne proviennent pas d'épaves.

Par ailleurs, nous constatons d'autres différences avec les échantillons archéologiques. Les teneurs en chlore de ces derniers sont inférieures aux limites de détection, alors qu'elles peuvent dépasser le pourcent pour les collections muséales. La présence du chlore peut par exemple être due à des chlorures, telles que des chlorures de sodium ou bien encore de calcium, des sels issus du milieu marin dans lequel pouvaient se trouver les céramiques ${ }^{18}$. Nous observons d'ailleurs une légère augmentation des teneurs en $\mathrm{Na}_{2} \mathrm{O}$ pour les pièces analysées en surface. L'un des autres éléments présentant globalement des teneurs plus élevées dans les pièces de musée est le fer avec des valeurs dépassant toutes 7,4\% mass. $\mathrm{Fe}_{2} \mathrm{O}_{3}$, à l'exception de la pièce MNC $22421^{5}$, contre $7,2 \%$ au maximum pour les échantillons analysés en coupe.

En raison de ces altérations, nous avons adapté notre méthodologie. De manière à pouvoir comparer les compositions des pâtes des céramiques issues des collections de Sèvres avec celles du groupe de référence de Chalcis, nous avons recalculé les compositions des premières de façon à nous affranchir des modifications chimiques les plus importantes résultant de phénomènes d'altération. Pour ce faire, les compositions ont été renormalisées à $100 \%$ sur les éléments majeurs et mineurs, sans intégrer les éléments $\mathrm{S}$ et $\mathrm{Cl}$, et en forçant la valeur de $\mathrm{MgO}$ à 2,5\% correspondant à la moyenne du groupe de référence de Chalcis (tableau 2).

\section{Origine des pièces}

En prenant en compte ces nouvelles compositions, nous avons vérifié au cas par cas si les pièces de musée pouvaient être rattachées à la production de Chalcis. Sur les neuf pièces analysées, deux présentent de très bonnes similitudes chimiques avec le groupe de référence. Il s'agit des pièces MNC 22896 et MNC $22421^{5}$ pour lesquelles les teneurs sont très proches de celles correspondant aux valeurs moyennes de référence. La céramique numérotée MNC 24782 témoigne aussi de bonnes concordances chimiques, sauf pour la valeur en $\mathrm{Na}_{2} \mathrm{O}$ bien plus élevée. Or cette céramique enregistre aussi la plus forte teneur en chlore, ce qui laisse supposer la présence de chlorure de sodium (sel marin). Ceci n'est pas surprenant compte tenu de l'état de surface de cette céramique riche en concrétions. En prenant en compte ce facteur, nous considérons aussi le plat MNC 24782 comme étant probablement issu de la production de Chalcis.

La pièce MNC $23415 \mathrm{Z} .1$ semble aussi pouvoir être rattachée à la production de Chalcis. Les compositions de la pâte de cette céramique sont en effet proches de celle du groupe de référence, à l'exception d'une valeur bien plus importante en phosphore $\left(1,08 \%\right.$ mass. $\mathrm{P}_{2} \mathrm{O}_{5}$, contre $0,19 \%$ pour la teneur moyenne de référence). Ces données chimiques résultant d'une analyse de surface, il est probable que nous ayons affaire à la présence de phosphates de calcium reflétant d'autres phénomènes d'altération causés par échanges ioniques lors de l'enfouissement de la céramique ${ }^{19}$; ce qui pourrait aussi expliquer la valeur un peu plus élevée en $\mathrm{CaO}$.

Les pièces MNC 26751, MNC 24950 et MNC 24783 présentent toutes des teneurs relativement élevées en titane et fer par rapport aux moyennes du groupe de référence, ce qui est aussi le cas pour l'échantillon archéologique BZY872 issu de l'épave de Kavalliani. Des teneurs en titane occasionnellement plus élevées avaient déjà été observées dans les céramiques de cette épave ${ }^{20}$. Quant aux valeurs en fer plus élevées observées sur la quasi-totalité des pièces de musée analysées, elles sont vraisemblablement elles aussi liées à une altération de surface en milieu marin. Nous pouvons noter que les teneurs en $\mathrm{SO}_{3}$ et $\mathrm{Cl}$ mesurées pour ces échantillons sont supérieures au pourcent, et la formation de pyrites dans les pâtes céramiques en milieu marin avait déjà été proposée $\mathrm{e}^{21}$. Il semblerait ainsi que cette combinaison de taux élevés en $\mathrm{Ti}, \mathrm{Fe}, \mathrm{S}$ et $\mathrm{Cl}$ soit le résultat d'altérations de surface, et qu'elle ne soit pas représentative de la pâte argileuse originelle ; ce qui nous laisse proposer une association de ces céramiques à la production de Chalcis.

\section{Discussion sur les objets douteux}

Enfin, deux pièces analysées - D SN 8 et MNC 24953 - se distinguent nettement des autres, notamment par leurs teneurs en calcium plus élevées (> $11 \%$ mass. CaO). Dans les deux cas, ces teneurs ne sont pas corrélées avec celles du soufre et du phosphore, ce qui exclut l'hypothèse de sulfates et phosphates de calcium formés durant l'enfouissement. La surface de la céramique D SN 8 ne présente aucune concrétion blanchâtre ; ce qui ne permet pas non plus d'expliquer cette teneur en calcium plus élevée par la formation de carbonates de calcium, tels l'aragonite, en milieu marin ${ }^{22}$. La teneur plus élevée en calcium et celle plus faible en aluminium de la céramique D SN 8 semblent donc bien propres à la composition originelle de sa pâte argileuse, et permettent de conclure que cette pièce ne provient pas de Chalcis.

Concernant la céramique MNC 24953, il est plus difficile de conclure. Sa surface est recouverte par endroit de concrétions blanchâtres, et bien que nous les ayons évitées ou éliminées en surface sur les zones analysées, il est possible que des cristaux secondaires de carbonates de calcium aient contribué aux teneurs en CaO. Par ailleurs, ce plat présente un style atypique par rapport au répertoire des céramiques de Chalcis. Ces différences sur le style et la composition chimique font douter de l'attribution de cette pièce à Chalcis.

Ainsi, sept des neuf pièces de musée analysées ont une bonne probabilité d'appartenir à la MBP et de provenir de Chalcis lato sensu. Il a été important de prendre en compte les phénomènes d'altération, d'autant plus qu'elles étaient analysées en surface, de façon non destructive. La céramique D SN 8 n'a, quant à elle, pas été produite par ce centre potier, ce qui va dans le sens de la mention « copie » sur la pièce, et le doute subsiste concernant le plat MNC 24953. 


\section{Analyses des glaçures}

Nous avons analysé les glaçures dans les mêmes conditions que les pâtes, à savoir en surface pour les plats de Sèvres et en coupe pour les échantillons archéologiques. L'étude des glaçures vise à définir en premier lieu leur nature, à savoir si elles sont plombifères, alcalino-plombifère ou bien alcalines. Toutes les glaçures analysées jusqu'à présent sur des céramiques byzantines à pâte rouge sont de type plombifère ${ }^{23}$; nous tâcherons notamment de vérifier si nous sommes toujours dans cette tendance.

Les compositions chimiques (tableau 3) des glaçures des collections de musée présentent, à l'exception de l'échantillon MNC 22896, des éléments pouvant être caractéristiques du milieu marin ou bien de mécanismes d'altération déjà évoqués pour les pâtes, que nous ne retrouvons pas dans les compositions des glaçures en coupe. Nous enregistrons en effet la présence des éléments $\mathrm{P}, \mathrm{S}$ et $\mathrm{Cl}$, ainsi que des teneurs nettement plus élevées en Ca.

Afin de comparer les analyses des glaçures des pièces de musée et des pièces archéologiques, nous avons utilisé une méthodologie analogue à celle mise en œuvre pour les pâtes. Les premières ont été recalculées et normalisées à $100 \%$ après soustraction des contributions des éléments $\mathrm{P}, \mathrm{S}, \mathrm{Cl}$ et $\mathrm{Ca}$ (ce dernier présentant des altérations peu marquées avec des valeurs inférieures à 1,7\% mass. CaO en coupe). Étant donné que la glaçure claire de la pièce MNC 26751 contient plus de $42 \%$ de ces éléments, sa composition, même recalculée, ne sera pas prise en compte car nous ne la considérons pas comme représentative.

Les compositions (recalculées) correspondent toutes à des glaçures plombifères avec des teneurs en $\mathrm{PbO}$ supérieures à $36 \%$ et de faibles teneurs en alcalins n'excédant pas les $4 \%$. Les colorants utilisés pour les décors peints ainsi que pour la coloration des glaçures sont de trois types. La couleur verte est obtenue par l'adjonction d'oxyde de cuivre dans la glaçure $(>2 \%$ mass. $\mathrm{CuO}$ ), tandis que les couleurs variant du jaune au brun sont dues à la présence du fer. Plus la teneur de ce dernier est élevée, plus la couleur est foncée, et passe du jaune au brun. Le troisième colorant que nous observons est à base de manganèse et de fer : il est utilisé pour le décor peint en brun foncé de l'échantillon BZY754.

Tableau 3. Compositions élémentaires des glaçures obtenues par analyses PIXE (en pourcentage massique d'oxyde ; *coul. gl. : couleur de la glaçure analysée $;<$ ld : valeur inférieure à la limite de détection).

\begin{tabular}{|c|c|c|c|c|c|c|c|c|c|c|c|c|c|c|c|}
\hline Échantillon / surface / coul. gl.* & $\mathrm{SiO}_{2}$ & $\mathrm{PbO}$ & $\mathrm{Na}_{2} \mathrm{O}$ & $\mathrm{K}_{2} \mathrm{O}$ & $\mathrm{MgO}$ & $\mathrm{CaO}$ & $\mathrm{Al}_{2} \mathrm{O}_{3}$ & $\mathrm{Fe}_{2} \mathrm{O}_{3}$ & CuO & $\mathrm{MnO}$ & $\mathrm{TiO}_{2}$ & $\mathrm{Sb}_{2} \mathrm{O}_{5}$ & $\mathrm{P}_{2} \mathrm{O}_{5}$ & $\mathrm{SO}_{3}$ & $\mathrm{Cl}$ \\
\hline \multicolumn{16}{|c|}{ Pièces de musée (analyse en surface) } \\
\hline MNC 26751 - bord externe orangé & 30.6 & 45.5 & 0.8 & 1.1 & 1.0 & 5.8 & 7.6 & 4.0 & 0.2 & 0.1 & 0.4 & 0.2 & 0.2 & 1.3 & 1.2 \\
\hline MNC 26751 - bord externe clair & 19.4 & 20.5 & 1.9 & 1.8 & 1.0 & 16.4 & 5.4 & 6.7 & 0.1 & 0.1 & 0.7 & $<$ ld & 0.3 & 15.4 & 10.4 \\
\hline MNC 224215 - interne vert & 39.6 & 43.0 & 0.8 & 2.8 & 0.8 & 2.1 & 4.9 & 0.8 & 1.9 & $<$ ld & 0.1 & 0.6 & 0.5 & 1.7 & 0.5 \\
\hline MNC 224215 - interne jaune & 30.5 & 46.9 & 0.3 & 2.1 & 0.5 & 6.3 & 3.8 & 1.0 & 0.4 & $<$ ld & 0.1 & 0.6 & 5.6 & 0.8 & 1.0 \\
\hline MNC 22896 - interne jaune clair & 28.1 & 65.3 & 0.4 & 1.0 & 0.3 & 0.8 & 2.8 & 0.7 & 0.1 & $<$ ld & $<$ ld & $<$ ld & $<$ ld & $<$ ld & 0.5 \\
\hline MNC 23415 - interne vert & 22.2 & 54.2 & 1.5 & 1.5 & 1.5 & 5.8 & 5.0 & 1.1 & 3.0 & 0.1 & 0.2 & $<$ ld & 0.6 & 1.1 & 2.3 \\
\hline \multicolumn{16}{|c|}{ Échantillons archéologiques (analyse en coupe) } \\
\hline BZY521 - interne incolore & 30.1 & 57.3 & 0.1 & 0.8 & 0.3 & 0.7 & 8.6 & 1.3 & 0.2 & $<$ ld & 0.2 & 0.4 & $<$ ld & $<$ ld & 0.1 \\
\hline BZY533 - interne brun & 38.5 & 37.1 & 0.4 & 1.7 & 1.0 & 0.8 & 14.3 & 5.6 & $<$ ld & $<$ ld & 0.3 & 0.1 & $<$ ld & $<$ ld & $<$ ld \\
\hline BZY533 - interne incolore & 41.6 & 44.2 & 0.3 & 1.3 & 0.4 & 0.7 & 10.4 & 0.8 & $<$ ld & $<$ ld & 0.2 & 0.1 & $<$ ld & $<$ ld & $<$ ld \\
\hline BZY754 - interne brun foncé / noir & 45.7 & 35.3 & 0.3 & 0.7 & 0.3 & 1.6 & 6.4 & 3.3 & 0.1 & 5.5 & 0.3 & $<$ ld & 0.2 & $<$ ld & 0.2 \\
\hline \multicolumn{16}{|c|}{ COMPOSITIONS RECALCULÉES APRÈS SOUSTRACTION DE P, S, CL ET CA } \\
\hline \multicolumn{16}{|c|}{ Pièces de musée (analyse en surface) } \\
\hline MNC 26751 - bord externe orangé & 33.4 & 49.7 & 0.9 & 1.2 & 1.1 & - & 8.3 & 4.4 & 0.2 & 0.1 & 0.4 & 0.3 & - & - & - \\
\hline MNC 224215 - interne vert & 41.5 & 45.1 & 0.8 & 3.0 & 0.9 & - & 5.2 & 0.8 & 2.0 & $<$ ld & 0.1 & 0.6 & - & - & - \\
\hline MNC 224215 - interne jaune & 35.3 & 54.3 & 0.3 & 2.5 & 0.6 & - & 4.4 & 1.2 & 0.5 & $<$ ld & 0.1 & 0.7 & - & - & - \\
\hline MNC 22896 - interne jaune clair & 28.4 & 66.1 & 0.4 & 1.0 & 0.3 & - & 2.8 & 0.7 & 0.1 & $<$ ld & $<$ ld & $<$ ld & - & - & - \\
\hline MNC 23415 - interne vert & 24.6 & 60.1 & 1.6 & 1.7 & 1.6 & - & 5.5 & 1.2 & 3.4 & 0.1 & 0.2 & $<$ ld & - & - & - \\
\hline \multicolumn{16}{|c|}{ Échantillons archéologiques (analyse en coupe) } \\
\hline BZY521 - interne incolore & 30.3 & 57.7 & 0.1 & 0.8 & 0.3 & - & 8.6 & 1.3 & 0.2 & $<$ ld & 0.2 & 0.4 & - & - & - \\
\hline BZY533 - interne brun & 38.8 & 37.4 & 0.4 & 1.7 & 1.0 & - & 14.4 & 5.7 & $<$ ld & $<$ ld & 0.3 & 0.1 & - & - & - \\
\hline BZY533 - interne incolore & 41.9 & 44.5 & 0.3 & 1.4 & 0.4 & - & 10.5 & 0.8 & $<$ ld & $<$ ld & 0.2 & 0.1 & - & - & - \\
\hline BZY754 - interne brun foncé / noir & 46.6 & 36.0 & 0.3 & 0.8 & 0.3 & - & 6.6 & 3.4 & 0.1 & 5.6 & 0.3 & $<$ ld & - & - & - \\
\hline
\end{tabular}


Ainsi, les glaçures analysées restent dans la tradition, déjà observée lors de précédentes études ${ }^{24}$, des glaçures plombifères appliquées sur les céramiques byzantines à pâte rouge. Cela se confirme aussi avec les colorants utilisés, qui permettent en effet d'obtenir les trois principales gammes de couleurs de ce type de céramiques byzantines. Il est intéressant de noter que la présence de manganèse a été mesurée uniquement dans une glaçure brun foncé d'une céramique à décor peint vert et brun ("Green and Brown Painted Ware"), ce qui correspond à ce qui avait déjà été observé par H. E. White sur des céramiques assimilées à la MBP découvertes à Corinthe ${ }^{25}$. La question de savoir si un pigment au manganèse était exclusivement appliqué sur ce type de décor peut alors se poser et mériterait d'être approfondie.

\section{Conclusion}

Les analyses menées par PIXE au C2RMF sur neuf céramiques mésobyzantines issues des collections du musée national de Céramique de Sèvres nous permettent de proposer l'attribution de sept d'entre elles à la production de Chalcis. Ces résultats ont été obtenus en utilisant une méthodologie adaptée à des pièces vraisemblablement issues d'épaves, qui présentent des altérations de leur composition chimique dues au milieu marin.

Nos résultats permettent en premier lieu de recontextualiser ces collections, et de mieux les restituer auprès du grand public. Cette démarche, encore trop rare, nous paraissait particulièrement importante pour faire bénéficier les collections muséales des toutes dernières avancées des recherches archéologiques et archéométriques. Par ailleurs, ils corroborent l'hypothèse selon laquelle les cargaisons de céramiques trouvées dans les épaves datées des XII ${ }^{\mathrm{e}}$ et XIII ${ }^{\mathrm{e}}$ siècles en mer Égée et en Méditerranée orientale proviendraient majoritairement de Chalcis ${ }^{26}$. Le fait que des pièces complètes soient souvent trouvées dans les épaves explique leur présence dans de nombreux musées ${ }^{27}$. Les glaçures appliquées sur ces céramiques sont plombifères, colorées dans les teintes vert, jaune-brun et brun foncé par l'adjonction respective d'oxydes de cuivre, de fer et de manganèse, ce qui concorde avec les traditions techniques jusqu'alors observées pour ces types de céramique.

\section{Remerciements}

Cette étude a été menée dans le cadre du programme ANR POMEDOR (People, Pottery and Food in the Medieval Eastern Mediterranean) financé par l'Agence Nationale pour la Recherche (référence ANR-12CULT-0008). Nous remercions Marie-Pierre Delanos, qui a rassemblé la documentation des pièces de musée étudiées lors de son stage effectué au musée national de Céramique de Sèvres ; Catherine Richarté (Inrap) pour sa contribution aux dessins de ces pièces, ainsi que Frédéric Guériel (Inrap) pour la DAO. Nous remercions aussi toute l'équipe d'AGLAE, dirigée par Claire Pacheco, pour leur aide lors de la réalisation des analyses. 
Notes

1. François, 2015 ; Koutsouflakis, à paraître.

2. Waksman, von Wartburg, 2006 ; Waksman et al., 2014, 2018 ; Waksman, 2018.

3. Vogt, Bouquillon, 1996 ; Vogt

et al., 1997; Lauffenburger et al., 2001 ; Bouquillon, 2017.

4. Papanikola-Bakirtzis, 1999, p. 145-149.

5. Cette pièce est actuellement en prêt au Louvre Abu Dhabi.

6. Conservés à l'Éphorie

des antiquités sous-marines d'Athènes pour les tessons BZY872 et BZI873, initialement conservés dans les éphories de Thèbes (BZY521, BZY533) et Chalkida (BZY754).

7. Wavelength-Dispersive X-Ray Fluorescence.

8. Waksman et al., 2018 ; Koutsouflakis, Tsompanidis, 2019

9. Waksman et al., 2014

10. Pichon et al., 2014.

11. Waksman et al., 2014.

12. Les comparaisons portant sur un corpus beaucoup plus important analysé dans les mêmes conditions sont en cours.

13. Béarat, 1990 ; Pradell et al., 1996 ;

Waksman et al., 2018, p. 1125 pour une bibliographie.

14. Waksman et al., 2018.

15. Pradell et al., 1996, p. 53.

16. Pradell et al., 1996, p. 55.

17. Pradell et al., 1996, p. 55.

18. Pradell et al., 1996, p. 49.

19. Freestone, 2001, p. 621.

20. Waksman et al., 2018.

21. Pradell et al., 1996, p. 54.

22. Pradell et al., 1996, p. 55. 2017.

23. Voir bibliographie dans Burlot,

24. Armstrong et al., 1997 ; White, 2009. 25. White, 2009, vol. 2, p. 268, tab. 8.4.7.

26. Waksman et al., 2014, 2018 ;

Waksman, 2018.

27. François, 2015 ; Waksman, 2018.

\section{Bibliographie}

Armstrong P., Hatcher H., Tite M. S., 1997, "Changes in Byzantine glazing technology from ninth to thirteenth centuries", dans Démians d'Archimbaud G. (dir.), Actes $d u V I^{\mathrm{e}}$ Congrès international sur la Céramique médiévale en Méditerranée (Aix-en-

Provence, 13-18 novembre 1995), Narrations éditions, Aix-en-Provence, p. 225-229.
Bouquillon A., 2017, « Analyses chimiques de quelques productions céramiques byzantines des collections du Louvre " dans François V., La vaisselle de terre à Byzance. Catalogue des collections du musée du Louvre, RMN Éditions, Paris, p. 310329.

François V., 2015, « De la cale à l'atelier : la vaisselle byzantine de la donation Janet Zakos au musée d'Art et d'Histoire de Genève ", dans Martiniani-Reber M. (dir.), Donation Janet Zakos: de Rome à Byzance, 5 Continents éditions, Genève, p. 201-217.

Freestone I. C., 2001, "Post-depositional Changes in Archaeological Ceramics and Glasses", dans Brothwell D. R. et Pollard A. M. (eds.), Handbook of Archaeological Sciences, Wiley, New York, p. 615-625.

Koutsouflakis G., Tsompanidis A., 2019, "The Kavalliani Shipwreck: A New Cargo of Byzantine Glazed Tableware from the South Euboean Gulf, Aegean", dans Yenişehirlioğlu F. (dir.), Proceedings of the $11^{\text {th }}$ International Congress AIECM3 on Medieval and Modern Period Mediterranean (Antalya, 19-24 October 2015), Koc University VEKAM, vol. 1, Ankara, p. 39-48.

Koutsouflakis G., à paraître, "The Transportation of Amphorae, Tableware and Foodstuffs in the Middle and the Late Byzantine Period: the Evidence from Aegean Shipwrecks", dans Waksman S. Y. (ed.) Multidisciplinary Approaches to Food and Foodways in the Medieval Eastern Mediterranean, Travaux de la Maison de l'Orient et de la Méditerranée, Lyon.

Lauffenburger J. A., Vogt C., Bouquillon A., 2001, "Technical Insights into the Working Practices of the Byzantine Tile Maker", dans Gerstel S. E. J. et Lauffenburger J. A. (eds.), A Lost Art Rediscovered. The Architectural Ceramics of Byzantium, Pennsylvania State University Press, Baltimore, p. 67-87.

Papanikola-Bakirtzis D., 1999, Byzantine Glazed Ceramics. The Art of Sgraffito, Museum of Byzantine Culture, Athens.

Pichon L., Moignard B., Lemasson Q, Pacheco C., Walter P., 2014,

"Development of a multi-detector and a systematic imaging system on the AGLAE external beam", Nuclear Instruments and Methods in Physics Research B, 318, p. 27-31.

Pradell T., Vendrell-Saz M., Krumbein W. E., Picon M., 1996, « Altérations de céramiques en milieu marin : les amphores de l'épave romaine de la Madrague de Giens (Var) ", Revue d'Archéométrie, 20, p. 47-56.
Vogt C., Bouquillon A., 1996, « Technologie des plaques murales décorées de Preslav et de Constantinople (IX ${ }^{\mathrm{e}}-\mathrm{XI}^{\mathrm{e}}$ siècles) ", Cahiers archéologiques, 44, p. 105-116.

Vogt C., Bouquillon A., Dubus M., Querré G., 1997, "Glazed wall tiles of Constantinople: physical and chemical characterization, manufacturing, and decorative processes", dans Maguire $\mathrm{H}$. (ed.), Materials Analysis of Byzantine Pottery, Dumbarton Oaks, Washington D.C., p. 51-65.

Waksman S. Y., von Wartburg M.-L., 2006, 'Fine Sgraffito Ware', 'Aegean Ware' and other Wares: new evidence for a major production of Byzantine ceramics", Report of the Department of Antiquities of Cyprus, p. 369-388.

Waksman S. Y., Kontogiannis N. D., Skartsis S. S., Vaxevanis G., 2014, "The Main 'Middle Byzantine Production' and Pottery Manufacture in Thebes and Chalcis", The Annual of the British School at Athens, 109, p. 379-422.

Waksman S. Y., 2018, "Defining the Main 'Middle Byzantine Production' (MBP): Changing Perspectives in Byzantine Pottery Studies", dans Yenişehirlioğlu F. (dir.), Proceedings of the $11^{\text {th }}$ International Congress AIECM 3 on Medieval and Modern Period Mediterranean (Antalya, 19-24 October 2015), Koç University VEKAM, vol. 1, Ankara, p. 397-407.

Waksman S. Y., Koutsouflakis G., Burlot J., Courbe L., 2018, "Archaeometric investigations of the tableware cargo of the Kavalliani shipwreck (Greece) and into the role of the harbour of Chalcis in the Byzantine and Frankish periods", Journal of Archaeological Science : Reports 21, p. 1122-1129.

\section{Documents inédits}

Béarat A., 1990, Étude de quelques altérations physico-chimiques des céramiques archéologiques, thèse de doctorat, université de Caen, France.

Burlot J., 2017, Premières productions de céramiques turques en Anatolie occidentale: Contextualisation et études techniques, thèse de doctorat, université de Lyon 2, France.

White H. E., 2009, An investigation of Production Technologies of Byzantine Glazed Pottery from Corinth, Greece in the eleventh to thirteenth centuries, thèse de doctorat, université de Sheffield, Royaume-Uni. 